MEJS Middle East Journal of Science

Research Article

\title{
APPLICATION OF NEW ITERATIVE ALGORITHM FOR THE NUMERICAL SOLUTION OF NONLINEAR CONVECTION-DIFFUSION EQUATION WITH CONSTANT COEFFICIENTS
}

\author{
Falade K.I ${ }^{1}$ (1) Mustaphar M. ${ }^{2}$ \\ ${ }^{1,2}$ Kano University of Science and Technology, Department of Mathematics, Faculty of Computing and \\ Mathematical Sciences, P.M.B 3244 Wudil Kano State Nigeria. \\ Corresponding author: faladekazeem2016@kustwudil.edu.ng
}

\begin{abstract}
This paper presents computational procedures for the formulation of an algorithm based on the new iterative method (NIM) for the numerical solution of the nonlinear heat equation with constant coefficients. The newly formulated algorithm (NIA) was successfully described the relationship between convection and diffusion constants. Three test cases (prototype) are considered for the investigation of time distribution profiles in the heat equation other studies. The algorithm is easy, efficient, and suggests solving similar problems in physical sciences and engineering.
\end{abstract}

Keywords: convection-diffusion heat equation, new iterative method (NIM), new iterative algorithm (NIA), partial differential equations (PDEs), convection-diffusion constant coefficients

\begin{tabular}{lll}
\hline Received: & September 28, 2020 & Accepted: March 11, 2021 \\
\hline
\end{tabular}

\section{Introduction}

Multidimensional dynamical systems which occur in sciences and engineering are modeled in partial differential equations (PDEs), especially the convection-diffusion equation occur in several areas of engineering such as chemical engineering, mechanical engineering, and petroleum engineering. Scientific investigation of phenomena and mathematical models are enormous tools for quantitative description and derivation of numerical conclusions. These models are in most cases in form of partial differential equations (PDEs) and therefore the solution to these kinds of equations is of great importance to scientists, engineers, researchers, and other concerned individuals. Partial differential equations are often used to describe multidimensional dynamical systems in engineering and mathematical physics and for obtaining solutions to problems of derivative displacement, velocity, concentration, mass diffusivity, and others. PDEs describe a relation between a multivariable function and its partial derivatives [1]. In thermodynamics study, convection-diffusion equation is one of the most important partial differential equations occurs which is used to describe heat transfer in air conditional unit, water transfer in soil, the spread of solute in a liquid flowing through a tube, dispersion of tracers in porous media, dispersion of dissolved salts in groundwater and long-range transport of pollutants in the atmosphere [2]. 
The convection-diffusion equation describes a phenomenon that arises when physical quantities are transferred inside the heat chamber due to diffusion and convection/advection reactions [3].

In this paper, we consider a nonlinear one-dimensional convection-diffusion equation with constant coefficients of the form:

$$
\frac{\partial u}{\partial t}+\alpha\left(\frac{\partial u}{\partial x}\right)^{2}-\beta \frac{\partial^{2} u}{\partial x^{2}}=0 . \quad \alpha, \beta>0
$$

with initial condition

$$
u(x, 0)=f(x) .
$$

where $\alpha$ and $\beta$ are velocity components of the fluid in the directions of the axes at the point $(x)$ at time $t, \alpha=\frac{k}{\rho D_{\rho}}$ here $k$ is the constant of thermal conductivity, $\rho$ and $D_{\rho}$ are density and specific heat of the fluid at constant pressure respectively. The first derivative $\frac{\partial}{\partial t}$ describes the motion of the fluid and $u(x, t)$ denotes the concentration at time $t$ of position $x$ and $f(x)$ is a known function.

Obtaining analytical and numerical solutions to this evolution problem by setting suitable initial conditions is useful to examine the time and position at which the constant coefficients behave which eventually use to determine $u(x, t)$. The basic analytical technique to solve equation (1) involves reducing the equation to diffusion equation by eliminating the convection term by introducing some moving coordinates has been a very serious setback especially when initial and boundary conditions are introduced [4]-[6].

Computational and numerical techniques play a major role in understanding the physical phenomenon in many areas of applied mathematics because of the longstanding challenges facing in obtaining analytical solutions [7]. Accordingly, numerical techniques are implored to obtain approximate/analytical solutions of the ordinary differential equation (ODE) and partial differential equation (PDE). Author [3] presented numerical solutions of the 1D/2D advection-diffusion equation using the method of inverse differential operators (MIDO) and [2] used a new finite difference equations couple with a numerical scheme to solve and analyze the advection-diffusion equation with constant and variable coefficients.

In recent years, several numerical techniques have been developed by many authors such as the adomian decomposition method (ADM), variational iterative method (VIM), differential transform method (DTM), homotopy perturbation method (HPM), new iterative method (NIM), change of variable and integral transform technique (CVIT), exponential variable transformation (EVT), a two-step scheme (TSS), a stabilized finite element formulation (SFEM), A multiscale/stabilized finite element method (MSFEM) and just to mention a few [9]-[17]. The main objective of this paper is to formulate a fast and efficient algorithm to solve the nonlinear convection-diffusion equation with constant coefficients and while a reduction in time and computational length involve are reduce. We hereby propose five steps algorithm using Maple 18 software for the numerical solutions of Eq.(1)

This paper concerns the usage of NIA to investigate the convection-diffusion heat equation presented in Eq.(1) with conditions (2). Section 2, we present a new iterative method (NIM) and formulated a new iterative algorithm, section 3 presents the numerical examples using the NIM to solve the Eq.(1) with initial condition (2), results and its discussion are presented in section 4, finally, the conclusion is presented in section 5 . 


\section{The New Iterative Method (NIM)}

New iterative method (NIM) as a numerical technique for solving the non-linear functional equation of the form [9].

$$
u(\bar{x})=f(\bar{x})+N(u(\bar{x})) .
$$

where $N$ a nonlinear operator from a Banach space $B \rightarrow B, f(\bar{x})$ is a known function, and $\bar{x}=$ $\left(x_{1}, x_{2}, x_{3}, \ldots, x_{n}\right)$.

In order to obtain solution for Eq.(3), we have series solution of the form

$$
u(\bar{x})=\sum_{i=0}^{\infty} u_{i}(\bar{x})
$$

Consider a nonlinear operator of the right-hand side of Eq.(3) can be decomposed as follows

$$
N\left(\sum_{n=0}^{\infty} u_{i}(\bar{x})\right)=N\left(u_{0}\right)+\sum_{i=1}^{\infty}\left\{N\left(\sum_{j=0}^{i} u_{j}\right)-N\left(\sum_{j=0}^{i-1} u_{j}\right)\right\} .
$$

Substitute Eq. (4) and Eq. (5) into the Eq. (3) leads to

$$
\sum_{i=0}^{\infty} u_{i}(\bar{x})=f(\bar{x})+N\left(u_{0}\right)+\sum_{i=1}^{\infty}\left\{N\left(\sum_{j=0}^{i} u_{j}\right)-N\left(\sum_{j=0}^{i-1} u_{j}\right)\right\} .
$$

Recurrence relation is given by

$$
\left\{\begin{array}{c}
u_{0}=f \\
u_{1}=N\left(u_{0}\right) \\
\vdots \\
u_{m+1}=N\left(u_{0}+u_{1}+\cdots+u_{m}\right)-N\left(u_{0}+u_{1}+\cdots+u_{m-1}\right) . \\
m=1,2,3, \ldots
\end{array}\right.
$$

Then;

$$
\left(u_{1}+u_{2}+\cdots+u_{m+1}\right)=N\left(u_{0}+u_{1}+\cdots+u_{m}\right), \quad m=0,1,2,3, \ldots, p .
$$

and

$$
\sum_{i=0}^{p} u_{i}=f+N\left(\sum_{i=0}^{p} u_{i}\right)
$$

The $p$-term approximate solution Eq.(3) is given as

$$
u=u_{0}+u_{1}+\cdots+u_{p-1}
$$

\subsection{Formulation of five steps New Iterative Algorithm (NIA)}

In order to formulate five steps algorithm, we consider Eq.(1) and Eq.(2) couple with Eq.(3)Eq.(10) as follows: 


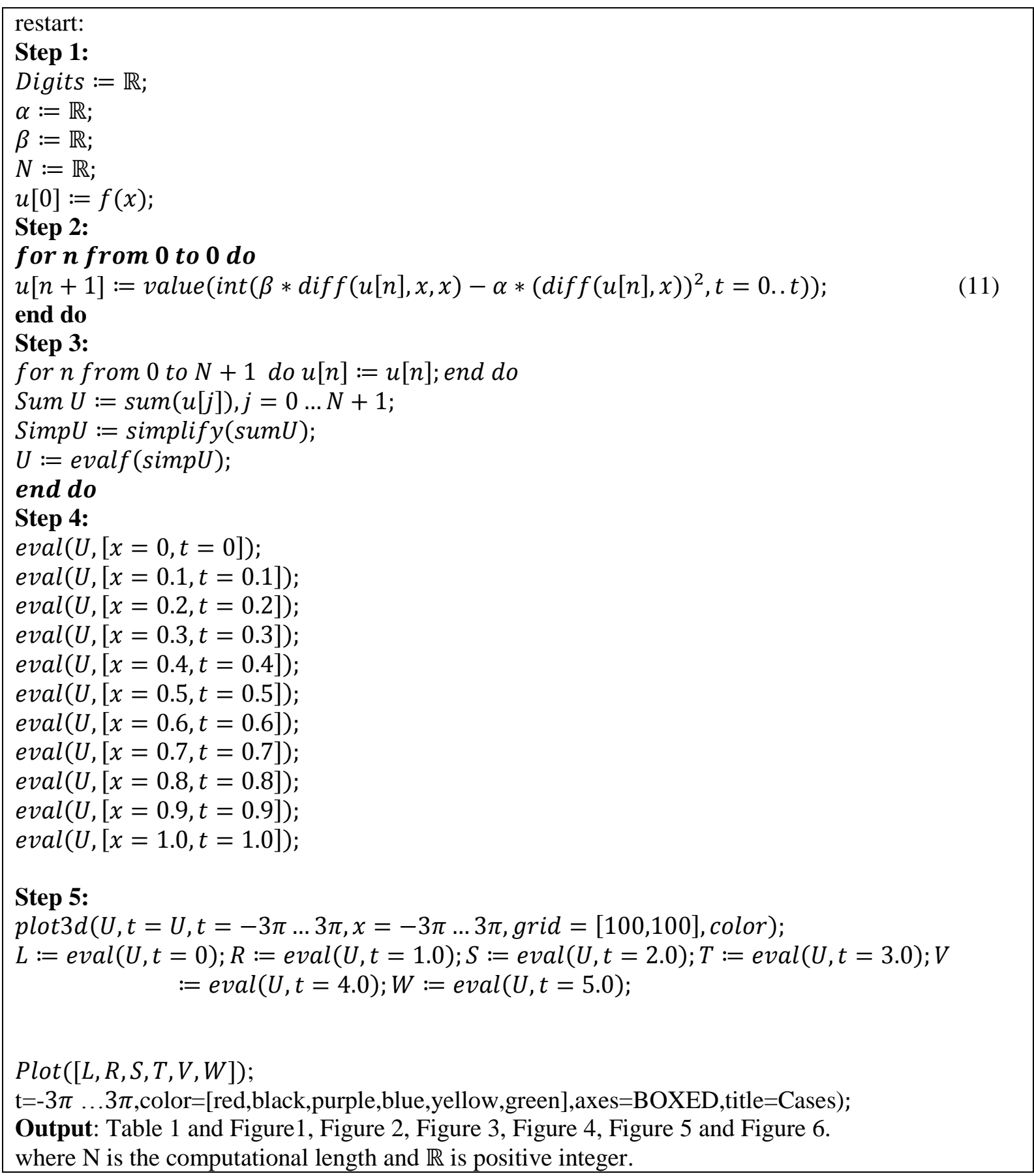

\subsection{Absolute error $\left(E_{t}\right)$}

To determine the error involved in the new iterative algorithm, we consider absolute error as follows:

$$
E_{t}=\left|\mathrm{u}(x, \mathrm{t})_{\text {exact }}-\mathrm{u}(x, \mathrm{t})_{\text {numerical }}\right| .
$$




\section{Computational experiment}

In this section, we apply a new iterative algorithm formulated in section 2.1 to solve and examine the behavior of advection-diffusion coefficients of heat equation of the form:

$$
\frac{\partial u}{\partial t}+\alpha\left(\frac{\partial u}{\partial x}\right)^{2}-\beta \frac{\partial^{2} u}{\partial x^{2}}=0
$$

with initial condition:

$$
u(x, 0)=f(x) . \quad 0 \leq x \leq 1
$$

$$
\text { where } f(x)=\exp \left(-\frac{1}{8}(x-2)^{2}\right) \text { and } \alpha=0.8, \beta=0.1
$$

\subsection{Numerical solutions}

\begin{tabular}{|c|c|c|c|c|}
\hline $\mathrm{u}(\mathrm{x}, \mathrm{t})$ & Solutions & $\begin{array}{c}\alpha=0.8, \beta=0.1 \\
\alpha>\beta\end{array}$ & $\begin{array}{c}\alpha=0.1, \beta=0.8 \\
\alpha<\beta\end{array}$ & $\begin{array}{c}\alpha=0.8, \beta=0.8 \\
\alpha=\beta\end{array}$ \\
\hline \multirow[t]{5}{*}{$(0,0)$} & Exact & 0.60653065971263342360 & 0.60653065971263342360 & 0.60653065971263342360 \\
\hline & NIA & 0.60653065971263342360 & 0.60653065971263342360 & 0.60653065971263342360 \\
\hline & DTM & 0.60653065971263342360 & 0.60653065971263342360 & 0.60653065971263342360 \\
\hline & $\mathrm{NIA}_{E_{t}}$ & 0.00000000000000000000 & 0.00000000000000000000 & 0.00000000000000000000 \\
\hline & $\mathrm{DTM}_{E_{t}}$ & 0.00000000000000000000 & 0.00000000000000000000 & 0.00000000000000000000 \\
\hline \multirow[t]{5}{*}{$(0.1,0.1)$} & Exact & 0.62939081883340116421 & 0.63451806527151677927 & 0.62835938510286210442 \\
\hline & NIA & 0.62939081883340116329 & 0.63451806527151677832 & 0.62835938510286210344 \\
\hline & DTM & 0.62939081883340116337 & 0.63451806527151677830 & 0.62835938510286210343 \\
\hline & $\mathrm{NIA}_{E_{t}}$ & 0.00000000000000000092 & 0.00000000000000000095 & 0.00000000000000000099 \\
\hline & $\mathrm{DTM}_{E_{t}}$ & 0.00000000000000000094 & 0.00000000000000000097 & 0.00000000000000000010 \\
\hline \multirow[t]{5}{*}{$(0.2,0.2)$} & Exact & 0.65207164215207057373 & 0.65967834907904235756 & 0.64797098393231306458 \\
\hline & NIA & 0.65207164215207057291 & 0.65967834907904235670 & 0.64797098393231306368 \\
\hline & DTM & 0.65207164215207057294 & 0.65967834907904235669 & 0.64797098393231306364 \\
\hline & $\mathrm{NIA}_{E_{t}}$ & 0.00000000000000000082 & 0.00000000000000000086 & 0.00000000000000000090 \\
\hline & $\mathrm{DTM}_{E_{t}}$ & 0.00000000000000000084 & 0.00000000000000000087 & 0.00000000000000000094 \\
\hline \multirow[t]{5}{*}{$(0.3,0.3)$} & Exact & 0.67462305112988452502 & 0.68205392686874213353 & 0.66541186559110849090 \\
\hline & NIA & 0.67462305112988452442 & 0.68205392686874213290 & 0.66541186559110849021 \\
\hline & DTM & 0.67462305112988452440 & 0.68205392686874213286 & 0.66541186559110849020 \\
\hline & $\mathrm{NIA}_{E_{t}}$ & 0.00000000000000000060 & 0.00000000000000000063 & 0.00000000000000000069 \\
\hline & $\mathrm{DTM}_{E_{t}}$ & 0.00000000000000000058 & 0.00000000000000000067 & 0.00000000000000000070 \\
\hline \multirow[t]{5}{*}{$(0.4,0.4)$} & Exact & 0.69707673326327307456 & 0.70170941422582969372 & 0.68067156308602757967 \\
\hline & NIA & 0.69707673326327307401 & 0.70170941422582969315 & 0.68067156308602757905 \\
\hline & DTM & 0.69707673326327307400 & 0.70170941422582969313 & 0.68067156308602757903 \\
\hline & $\mathrm{NIA}_{E_{t}}$ & 0.00000000000000000055 & 0.00000000000000000057 & 0.00000000000000000062 \\
\hline & $\mathrm{DTM}_{E_{t}}$ & 0.00000000000000000056 & 0.00000000000000000059 & 0.00000000000000000060 \\
\hline
\end{tabular}

Numerical results for $\alpha>\beta, \quad \alpha<\beta$ and $\alpha=\beta$ are presented in Table 1 .

Table 1. Numerical results when $\alpha>\beta, \alpha<\beta$ and $\alpha=\beta$ 
Table 1. continued

\begin{tabular}{|c|c|c|c|c|}
\hline \multirow[t]{5}{*}{$(0.5,0.5)$} & Exact & 0.71943675747359916324 & 0.71871949819843862207 & 0.69370630455972661309 \\
\hline & NIA & 0.71943675747359916289 & 0.71871949819843862170 & 0.69370630455972661267 \\
\hline & DTM & 0.71943675747359916287 & 0.71871949819843862168 & 0.69370630455972661265 \\
\hline & $\mathrm{NIA}_{E_{t}}$ & 0.00000000000000000033 & 0.00000000000000000037 & 0.00000000000000000042 \\
\hline & $\mathrm{DTM}_{E_{t}}$ & 0.00000000000000000035 & 0.00000000000000000039 & 0.00000000000000000044 \\
\hline \multirow[t]{5}{*}{$(0.6,0.6)$} & Exact & 0.74167226603122097657 & 0.73315908521491595694 & 0.70447662969211101240 \\
\hline & NIA & 0.74167226603122097625 & 0.73315908521491595660 & 0.70447662969211101212 \\
\hline & DTM & 0.74167226603122097624 & 0.73315908521491595659 & 0.70447662969211101210 \\
\hline & $\mathrm{NIA}_{E_{t}}$ & 0.00000000000000000032 & 0.00000000000000000034 & 0.00000000000000000038 \\
\hline & $\mathrm{DTM}_{E_{t}}$ & 0.00000000000000000033 & 0.00000000000000000035 & 0.00000000000000000040 \\
\hline \multirow[t]{5}{*}{$(0.7,0.7)$} & Exact & 0.76371288467368716254 & 0.74509607179104746658 & 0.71299349576292573991 \\
\hline & NIA & 0.76371288467368716226 & 0.74509607179104746627 & 0.71299349576292573955 \\
\hline & DTM & 0.76371288467368716225 & 0.74509607179104746628 & 0.71299349576292573957 \\
\hline & $\mathrm{NIA}_{E_{t}}$ & 0.00000000000000000028 & 0.00000000000000000031 & 0.00000000000000000036 \\
\hline & $\mathrm{DTM}_{E_{t}}$ & 0.00000000000000000029 & 0.00000000000000000033 & 0.00000000000000000038 \\
\hline \multirow[t]{5}{*}{$(0.8,0.8)$} & Exact & 0.78544698891154179220 & 0.75458681710980188921 & 0.71936470465768928581 \\
\hline & NIA & 0.78544698891154179195 & 0.75458681710980188895 & 0.71936470465768928564 \\
\hline & DTM & 0.78544698891154179195 & 0.75458681710980188896 & 0.71936470465768928565 \\
\hline & $\mathrm{NIA}_{E_{t}}$ & 0.00000000000000000025 & 0.00000000000000000026 & 0.00000000000000000027 \\
\hline & $\mathrm{DTM}_{E_{t}}$ & 0.00000000000000000025 & 0.00000000000000000027 & 0.00000000000000000028 \\
\hline \multirow[t]{5}{*}{$(0.9,0.9)$} & Exact & 0.80672244981892401518 & 0.76167416913697207089 & 0.72383206904870742665 \\
\hline & NIA & 0.80672244981892401497 & 0.76167416913697207065 & 0.72383206904870742638 \\
\hline & DTM & 0.80672244981892401497 & 0.76167416913697207066 & 0.72383206904870742640 \\
\hline & $\mathrm{NIA}_{E_{t}}$ & 0.00000000000000000021 & 0.00000000000000000024 & 0.00000000000000000027 \\
\hline & $\mathrm{DTM}_{E_{t}}$ & 0.00000000000000000021 & 0.00000000000000000023 & 0.00000000000000000025 \\
\hline \multirow[t]{5}{*}{$(1.0,1.0)$} & Exact & 0.82734908472124433329 & 0.76638773317667365521 & 0.72678998055516529030 \\
\hline & NIA & 0.82734908472124433312 & 0.76638773317667365504 & 0.72678998055516529009 \\
\hline & DTM & 0.82734908472124433311 & 0.76638773317667365502 & 0.72678998055516529009 \\
\hline & $\mathrm{NIA}_{E_{t}}$ & 0.00000000000000000019 & 0.00000000000000000021 & 0.00000000000000000021 \\
\hline & $\mathrm{DTM}_{E_{t}}$ & 0.00000000000000000018 & 0.00000000000000000019 & 0.00000000000000000021 \\
\hline
\end{tabular}

Where NIA $E_{t}$ Absolute error obtained for New Iterative Algorithm (NIA). DTM $_{E_{t}}$ Absolute error obtained for Differential Transformation Method (DTM).

\subsection{Plot representation}

The graphical results obtained for the different cases of $\alpha, \beta$ and logarithm of absolute errors are presented in Fig 1-9. 


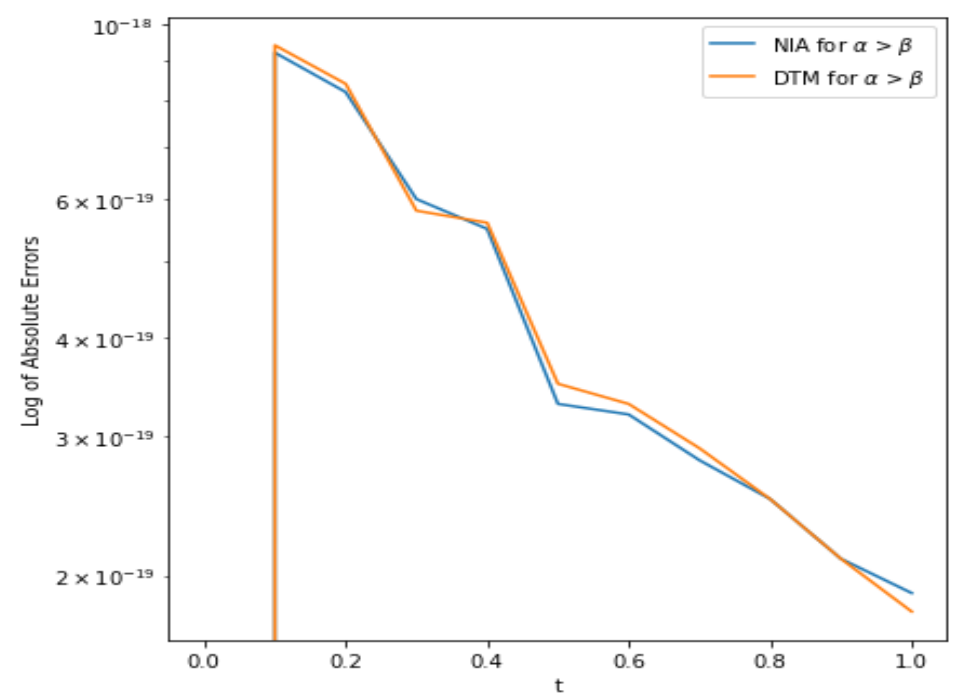

Figure 1: Logarithm of Absolute Errors for NIA and DTM for case 1

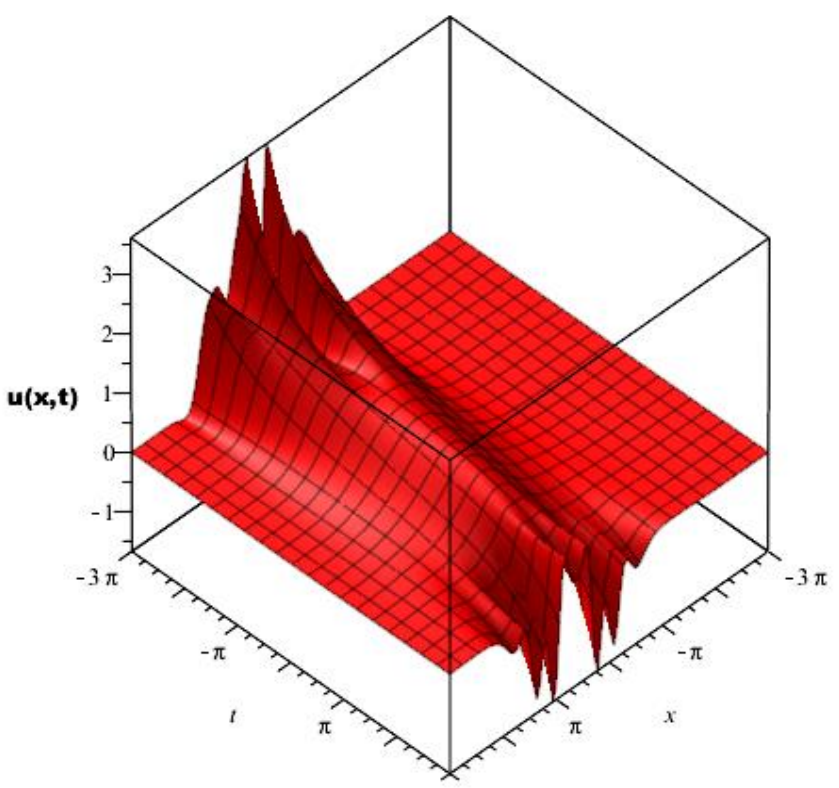

Figure 2. 3D-plot profiles when convection term is greater than diffusion term case $1 \alpha>\beta$ 


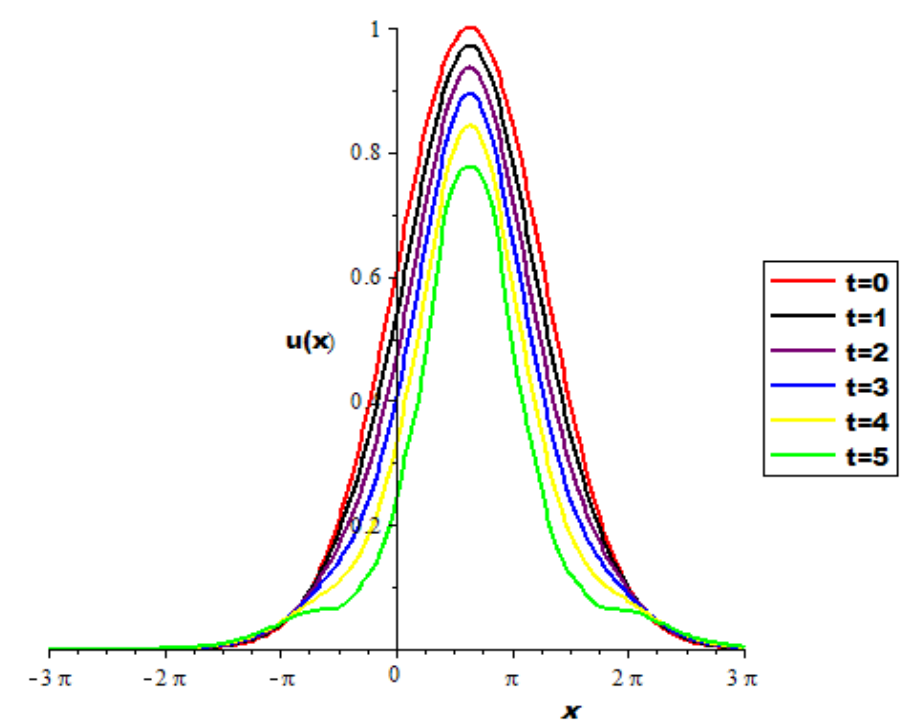

Figure 3. 2D-plot for time distribution profiles from initial time $0 \mathrm{sec}$ to $5 \mathrm{sec}$ when convection term is greater than diffusion term case $1 \alpha>\beta$

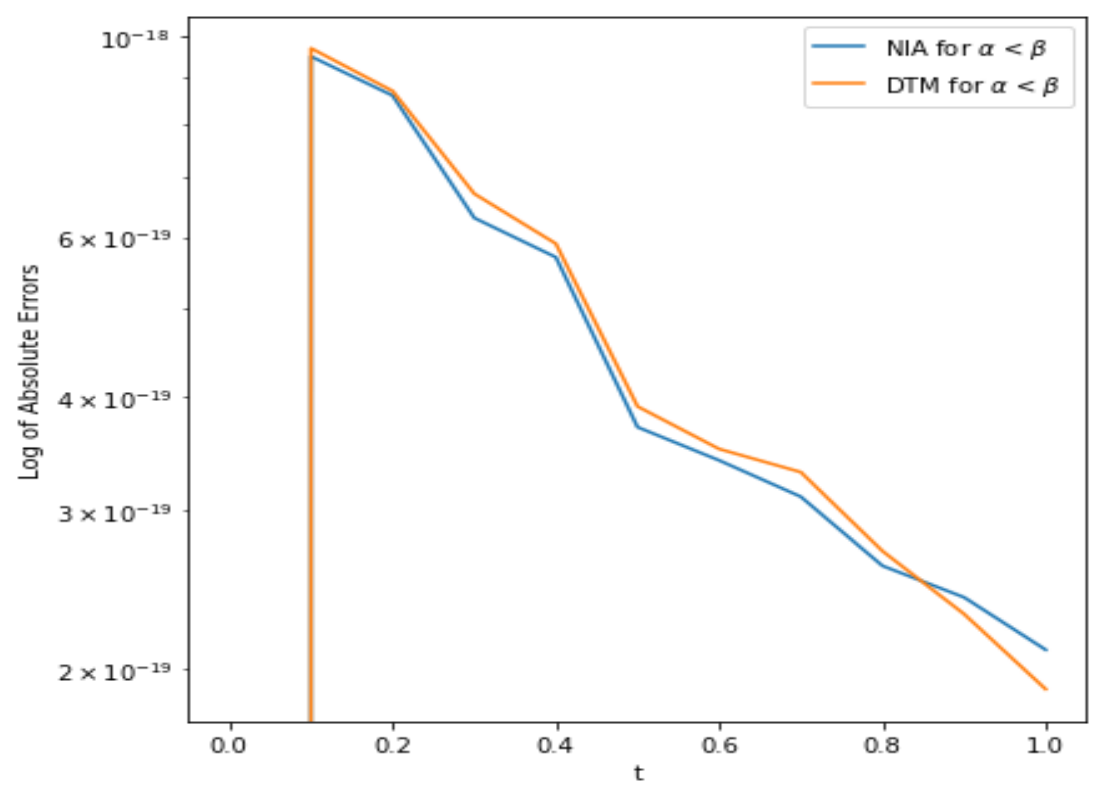

Figure 4: Logarithm of Absolute Errors for NIA and DTM for case 2 


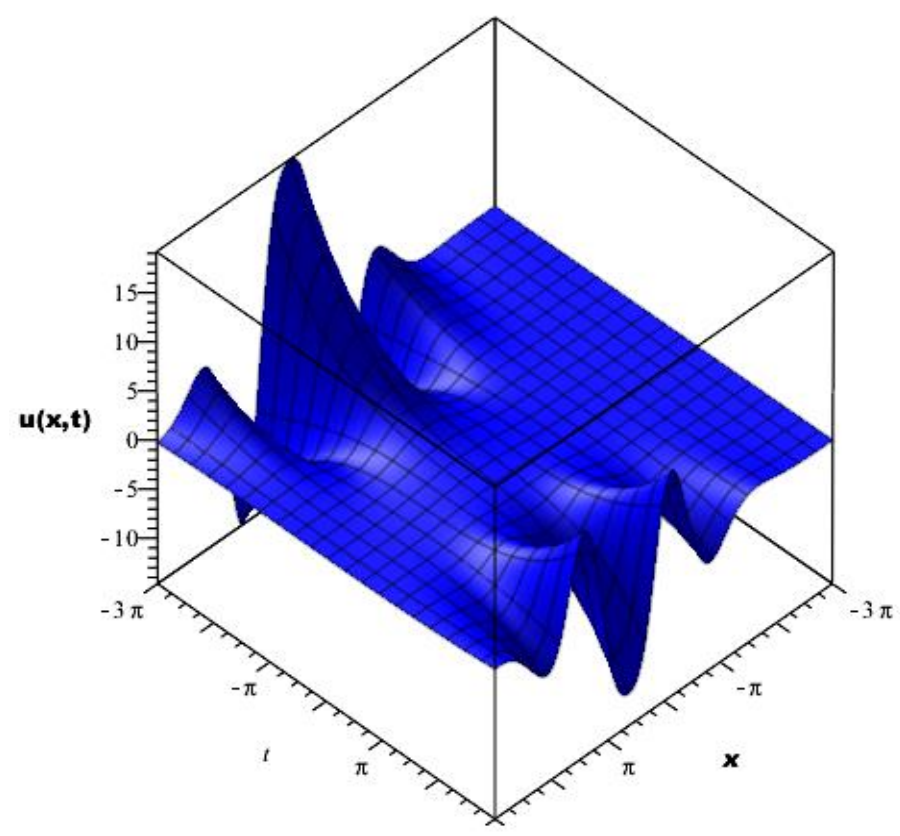

Figure 5. 3D-plot profiles when convection term is less than diffusion term case $2 \alpha<\beta$.

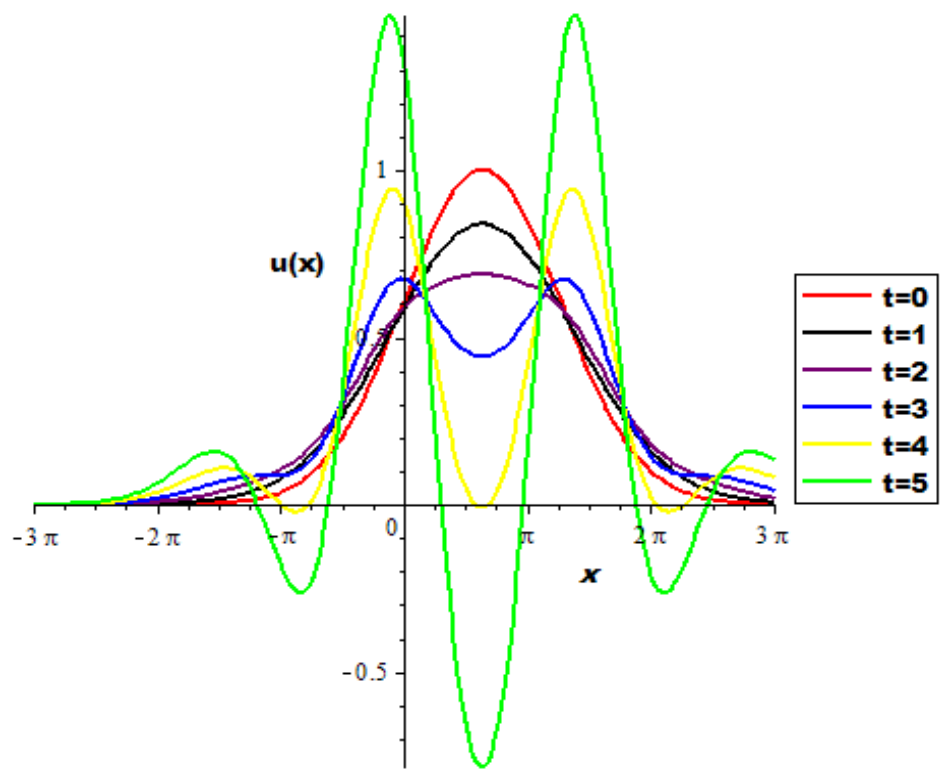

Figure 6. 2D-plot for time distribution profiles from initial time $0 \mathrm{sec}$ to $5 \mathrm{sec}$ when convection term is less than diffusion term case $2 \alpha<\beta$ 


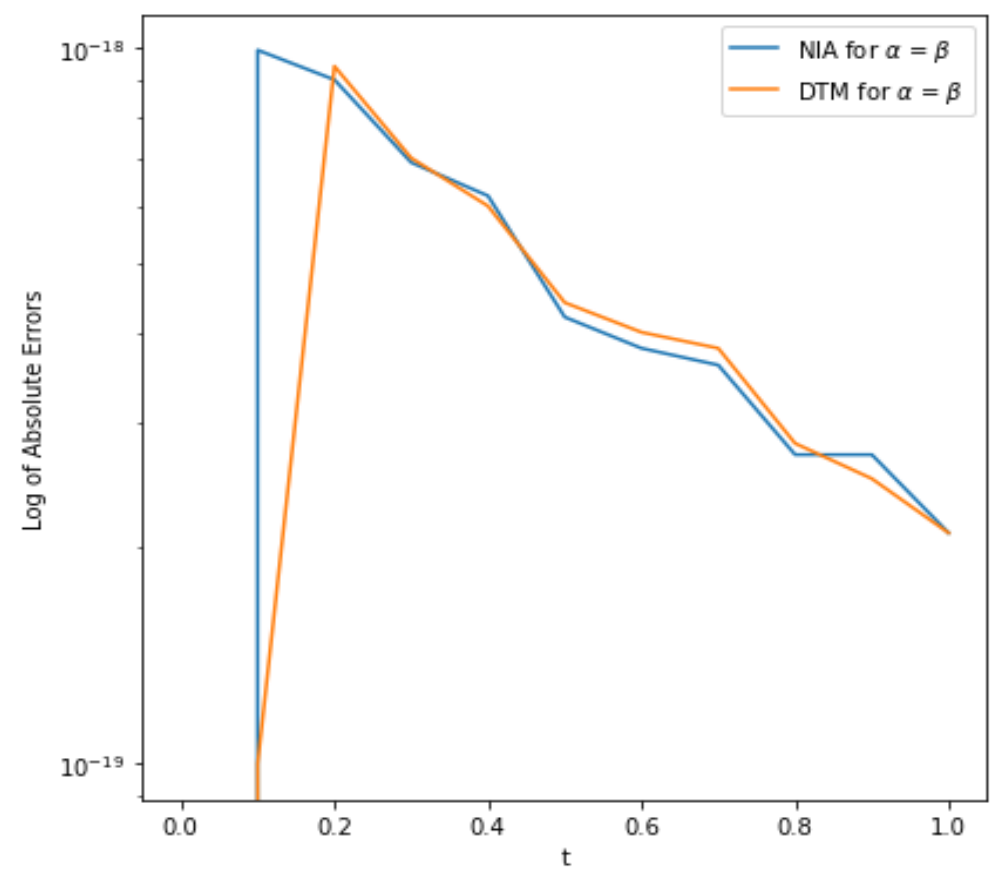

Figure 7: Logarithm of Absolute Errors for NIA and DTM for case 3

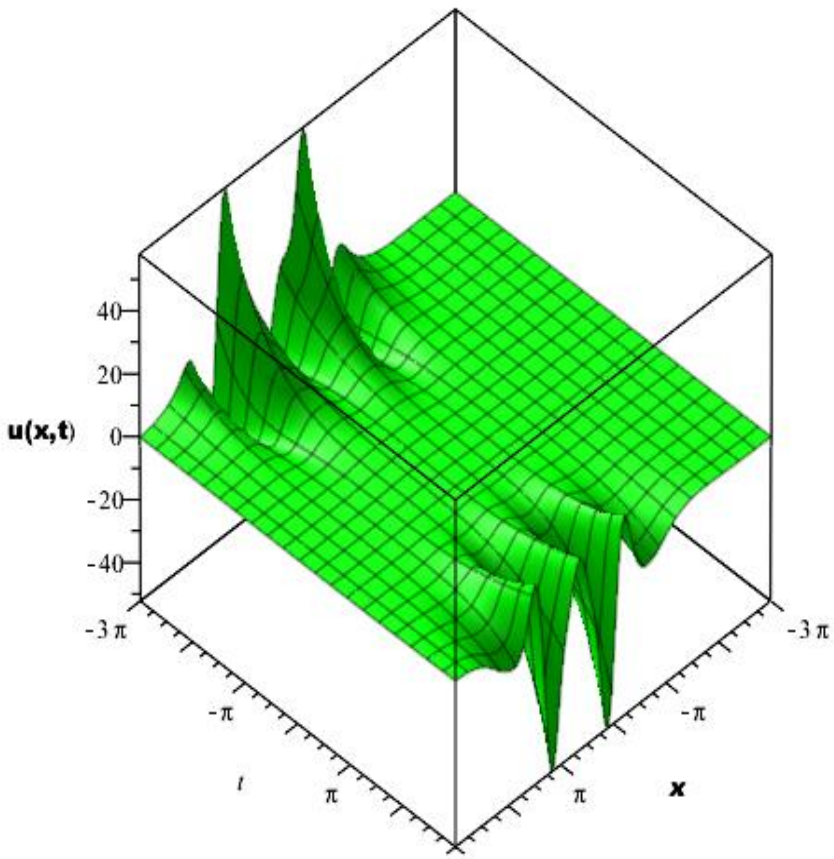

Figure 8. 3D-plot profiles when convection term is equal to diffusion term case $3 \alpha=\beta$. 


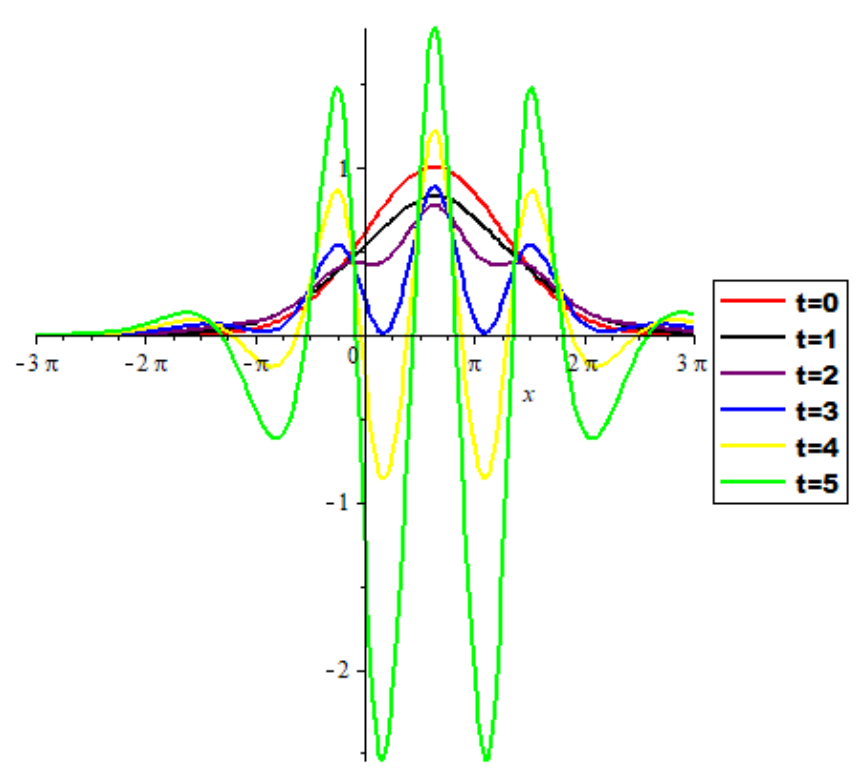

Figure 9. 2D-plot for time distribution profiles from initial time $0 \mathrm{sec}$ to $5 \mathrm{sec}$ when convection term is equal to diffusion term case $3 \alpha=\beta$.

\section{Discussion}

In this paper, we examine the numerical relationship and effect of convection $\alpha$ and diffusion $\beta$ constant coefficients which serve as velocity components of the fluid in the directions of the axes at the point $(x)$ at time $t$ of Eq.(3). Table 1 shows numerical solutions obtained for three experimental cases considered (when convection constant is greater than diffusion constant $\alpha>\beta$, convection constant is less than diffusion constant $\boldsymbol{\alpha}<\boldsymbol{\beta}$ and convection constant are equal to diffusion constant $\boldsymbol{\alpha}=\boldsymbol{\beta}$ ). From computational solutions obtained, we observe the following:

i. Increases in numerical solutions $u(x, t)$ are obtained when the convection constant is greater than diffusion constant $\alpha>\beta$.

ii. Less numerical solutions $u(x, t)$ were obtained when the convection constant is equal to diffusion constant $\boldsymbol{\alpha}=\boldsymbol{\beta}$.

Furthermore, Figures 1,4 and 7 depict the pertain of absolute errors in logarithm when compare the two numerical techniques presented (NIA and DTM) with exact solutions while figures 2, 5, and 8 show the 3D-plots of heat distribution solution for the two constant coefficients $\boldsymbol{\alpha}$ and $\boldsymbol{\beta}$ and the Figures 3,6 and 9 show 2D-plots that depict the time distributions profiles from initial time $0 \mathrm{sec} \leq t \leq 5 \mathrm{sec}$ and the following observations are deduced:

i. Reverse time distribution profiles were obtained at $\boldsymbol{\alpha}>\boldsymbol{\beta}(0 \mathrm{sec} \leq t \leq 5 \mathrm{sec})$.

ii. Oscillating and hypergeometric distribution occurred at $5 \sec$ (green) when $\boldsymbol{\alpha}<\boldsymbol{\beta}$ and $\boldsymbol{\alpha}=\boldsymbol{\beta}$.

iii. Minimum heat distribution occurred at $5 \sec$ (green) when $\boldsymbol{\alpha}>\boldsymbol{\beta}$.

iv. Non-uniform distribution heat profiles occurred at $\mathbf{1} \boldsymbol{s e c} \leq \boldsymbol{t} \leq \mathbf{4} \boldsymbol{s e c}$ (black, purple, blue, and yellow).

\section{Conclusion}

The formulated algorithm was successfully applied to solve nonlinear convection and diffusion heat equations with constant coefficients. Three test cases (prototype) are considered to demonstrate the feasibility and efficiency of the proposed algorithm. From the computational point of view, the new 
iterative algorithm (NIA) obtained fewer errors compared to the differential transformation method (DTM). Moreover, the main advantage of NIA is its simplicity with small computational costs and faster convergence. The present approach is very reliable, simple, fast, and convenient. Thus, we hereby suggest NIA as a good numerical technique to solve similar problems in applied mathematics and engineering sciences.

\section{The compliance to Research and Publication Ethics}

This work was carried out by obeying research and ethics rules.

\section{Conflict of interest}

The authors declare no conflict of interest.

\section{Acknowledgments}

The authors wish to express their gratitude to the editorial board and reviewers for their reading of this manuscript and helpful suggestions.

\section{Authors' Contributions}

Falade K.I: Conceptualization, designed the methodology and performed the computational analysis (60\%), Mustaphar M: Managed the analysis of the study, vetting the literature searches and typing the article $(40 \%)$ and both approved the final manuscript.

\section{References}

[1] M. C. Methods, "Solving nonlinear and high-dimensional partial differential equations via deep learning”, EMAp, Fund. Getulio Vargas, Rio Janeiro, Brazil, vol. I, 2019.

[2] S. G. Ahmed, "A numerical algorithm for solving advection-diffusion equation with constant and variable coefficients", The open Numerical Methods Journal, 3, 59-65, 2011.

[3] R. Kragler, "Solution of 1D/2D advection-diffusion equation using the method of inverse differential operators (MIDO) ", Conference Paper Weingarten University of Applied Sciences D-88241 Weingarten, Germany pp1-15, 2015.

[4] A. Kumar, D. K. Jaiswal, and N. Kumar, "Analytical solutions of one-dimensional advection diffusion equation with variable coefficients in a finite domain", Journal of Earth System Science, 118, 539-549, 2009.

[5] D. K Jaiswal, A. Kumar, R. R. Yadav "Analytical Solution to the One-Dimensional AdvectionDiffusion Equation with Temporally Dependent Coefficients", Journal of Water Resource and Protection, 3, 76-84, 2011.

[6] A. Peirce, "Heat conduction with time-dependent boundary conditions using eigen-function expansions", Introductory lecture notes on partial differential equations, 1-9, 2020.

[7] J. Biazar and H. Ghazvini, "Homotopy perturbation method for solving hyperbolic partial differential equations", Computer and Math. With Appl., 56(2), 453-458, 2008.

[8] M. Dehghan, "On the numerical solution of the one-dimensional convection-diffusion equation”, Hindawi Publishing Corporation Mathematical Problems in Engineering, 61-74, (1)2005. 
[9] M. Yaseen and M. Samraiz, "The modified new iterative method for solving linear and nonlinear Klein Gordon equations new iterative method", Applied Mathematical Sciences, 6(60), $2979-2987,2012$.

[10] R. Behl, A.Cordero, J. R. Torregrosa and A. S. Alshomrani "new iterative methods for solving nonlinear problems with one and several unknowns”, MDPI sum Mathematics, 6, 296, 2018.

[11] S. Bhalekar, and V. Gejji, "new iterative method: application to partial differential equations", Applied mathematics and computation, 203, 778-783, 2008.

[12] C. Chun, "A new iterative method for solving nonlinear equations", Applied mathematics and computational, 178, 415-422, 2006.

[13] J. S. Pérez Guérrero, L. C. G. Pimentel, T. H. Skaggs, M. Th. VanGenuchten, "Analytical solution of the one-dimensional advection-diffusion transport equation using a change of variable and integral transform technique", Int. J. Heat and Mass Transfer, 52, 3297-3304, 2009.

[14] K.M Singh, M. Tanaka "On exponential variable transformation based boundary element formulation for advection-diffusion problems", Eng anal bound element, 24, 225-35, 2000.

[15] L.L.Takacs, "A two-step scheme for the advection equation with minimized dissipation and dispersion errors", Monthly Weather Review, 113(6), 1050-1065, 1985.

[16] D. Z. Turner, K. B. Nakshatrala and K. D. Hjelmstada "Stabilized finite element formulation for advection-diffusion using the generalized finite element framework", https://www.researchgate 2008.

[17] A. Masud and R. A. Khurram. "A multiscale/stabilized finite element method for the advection- diffusion equation", Computer Methods in Applied Mechanics and Engineering, 193, 1997-2018, 2004. 